\title{
S100B and neuron specific enolase (NSE) value for prediction of in-hospital mortality for patients with severe traumatic brain injury
}

\author{
$\underline{\text { A.Vilke }}^{1,2}$, D. Bilskiene'2, V. Traskaite², D. Ranceviene' ${ }^{2}$ A. Macas² \\ ${ }^{1}$ Lithuanian University of Health Sciences, Department of Anesthesiology, Neuroscience Institute, Kaunas, Lithuania \\ ${ }^{2}$ Lithuanian University of Health Sciences, Dept. of Anesthesiology, Kaunas, Lithuania
}

\begin{abstract}
Background and Aim of the Study
Measurement of neuromarkers is easy but not widely used diagnostic method. The value of neuromarkers for predicting the outcome of traumatic brain injury (TBI)

is not clear enough. The aim of this study was to evaluate the value of S100B and NSE for prediction of

in-hospital mortality for patients with severe TBI.
\end{abstract}

Figure 1. ROC curves of S100B and NSE values 24 and 48 hours after admission (lower)
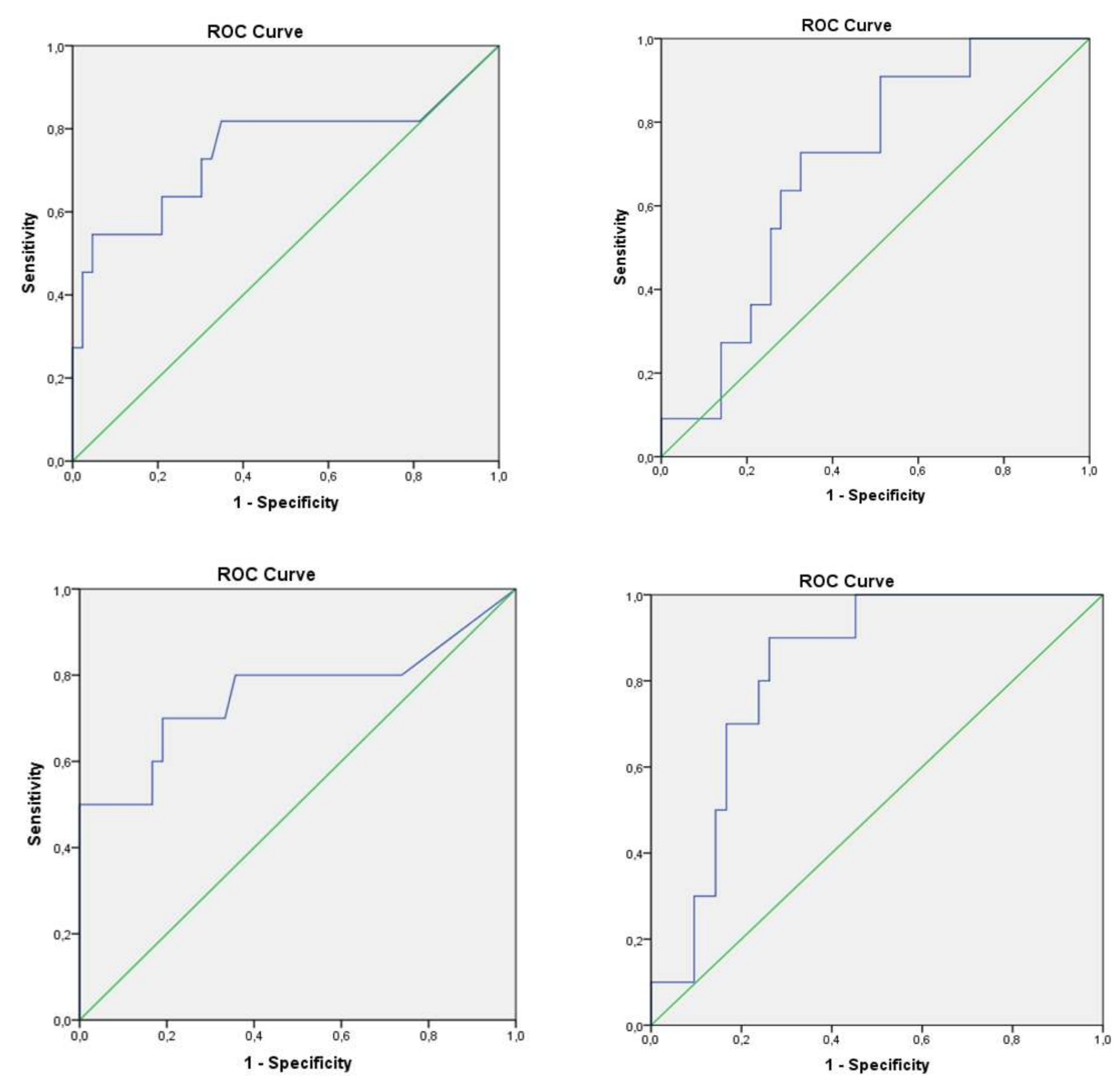

\section{Materials and Methods}

A prospective study was held in Lithuanian University of Health Sciences Kaunas Clinics, Anesthesiology clinic. Serum of 46 patients with severe TBI was analyzed for S100B and NSE neuromarkers. Neuromarkers were measured at hospital admission (N1) and after 24 (N2), 48 (N3) and 72 (N4) hours.

Outcomes were analyzed at hospital discharge and the

influence of neuromarkers for in-hospital mortality was

evaluated using ROC curves. Patients with absence of measurements were excluded from the study. Nonparametric tests were used for statistical analysis at $p \leq 0.05$. Approval of Regional bioethics committee was obtained before study initiation.

\section{Results}

46 patients were involved into the study. There were 11 women and 35 men. The average age was $55.33 \pm 16.8$ years and there was no significant difference between women and

men. Statistically significant differences were found comparing N1-N3, N1-N4, N2-N4, N3-N4 measurements of S100B and N1-N2, N1-N3, N1-N4, N2-N4 measurements of NSE.

Using ROC curves analysis specific values of breaking points were found for in-hospital mortality prediction. In-hospital mortality was significantly higher when S100B was N1 >22.5 $\mathrm{pg} / \mathrm{ml}, \mathrm{N} 2>128.5 \mathrm{pg} / \mathrm{ml}, \mathrm{N} 3>27.04 \mathrm{pg} / \mathrm{ml}, \mathrm{N} 4>112.06 \mathrm{pg} / \mathrm{ml}$ and NSE was N2 >7.97 $\mu \mathrm{g} / \mathrm{l}, \mathrm{N} 3>5.8 \mu \mathrm{g} / \mathrm{l}, \mathrm{N} 4>28.6 \mu \mathrm{g} / \mathrm{l}$. N1

measurement of NSE had no statistically significant difference.

Statistically significant difference among the survivors and inhospital mortality was found in N1, N2, N3 of S100B

measurements and in N2, N3 of NSE measurements.

Moreover, S100B values N2 $>128.5 \mathrm{pg} / \mathrm{ml}$ and N3 $>27.04$ $\mathrm{pg} / \mathrm{ml}$ increased the risk of in-hospital mortality 18.174 and

7.67 times respectively as well as NSE N2 $>7.97 \mu \mathrm{g} / \mathrm{l}$ and N3 $>5.8 \mu \mathrm{g} / \mathrm{l}$ increased the risk 5.4 and 21.671 times respectively.

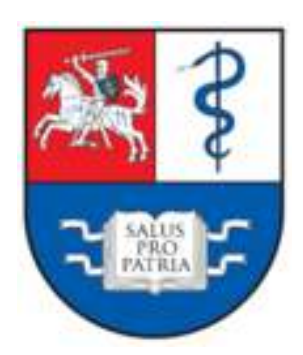

\section{Conclusion}

S100B and NSE can be used for prediction of in-hospital mortality for severe TBI patients. However, the maximum value for prediction can be obtained if neuromarkers are measured after 24 and 48 hours after hospital admission.

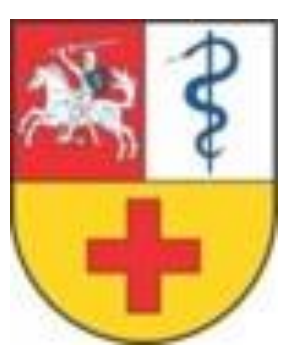

